\title{
Implementation of Precaptured Videos of Thyroid Fine- Needle Aspiration Cytology Specimens for External Quality Control Purposes
}

\author{
Archondakis S* \\ Department of Cytopathology, Alpha Prolipsis \\ Cytopathology Laboratories, Greece \\ *Corresponding author: Stavros Archondakis, \\ Department of Cytopathology, Alpha Prolipsis \\ Cytopathology Laboratories, 1Xenias St, Athens, 12137, \\ Greece
}

Received: December 31, 2020; Accepted: J anuary 12, 2021; Published: J anuary 19, 2021

\begin{abstract}
Objective: The objective of this study was examine the feasibility of developing a proficiency testing scheme for cytology labs wishing to be accredited according to ISO 15189:2012, by using videos captured by static telecytological applications.

Methods: The current study was carried out on 252 adequate specimens of 157 patients, retrospectively selected from the department's registry. In all cases, surgical excision followed the initial cytological diagnosis. Three diagnostic categories of cytological reports were used. All cases were confirmed by histological diagnosis of surgical specimens. One representative video from each case was transferred via file transfer protocol to password-protected accounts for remote review by 3 independent cytopathologists. In addition to diagnosis, reviewers commented on overall digital video quality. Contributor's and reviewer's diagnoses were collected, recorded and statistically evaluated.
\end{abstract}

Results: Statistical evaluation of cytological diagnoses detected no significant difference in diagnostic accuracy between the diagnoses proffered on the basis of precaptured videos and conventional slides. The overall interobserver agreement was ranging from substantial to almost perfect.

Conclusions: Videos production by static telecytology applications can be used as an alternative method for cytological diagnosis validation. It is a prompt and valid method for quality assessment and proficiency testing and can be integrated into daily workflow. Pre-captured videos can improve significantly small cytology departments' quality indices. Precaptured videos can also be used for teleconsultation and second opinion purposes and improve the performance of the already existing static telecytology stations.

Keywords: Telecytology; Liquid-based cytology; Thyroid; Accreditation; Quality control; Fine-needle aspiration; Videos

\section{Introduction}

Accreditation is the process by which a certified organization or agency recognizes that a facility or service meets specific preestablished standards. ISO 15189:2012 constitutes an international accreditation standard, which can be used by medical laboratories wishing to improve their quality standards. Its requirements consist of a group of general guidelines that will help laboratories establish and enhance their quality systems [1-4]. According to ISO 15189:2012 requirements, one of the greatest challenges facing cytology laboratories is the design and implementation of a board certified external quality control program [1-4]. The purpose of the adopted program should be to ensure that microscopic (cytological) findings are correctly identified and interpreted by laboratory personnel [1-3]. Telecytology is the interpretation of cytology material at a distance using digital material (images or videos) [5-8]. Telecytology can be used for teaching, professional assessment, auditing, archiving, quantitative cytology and research, obtaining expert opinions on difficult cases, and routine diagnosis of the entire laboratory workload [5-7]. Telecytological diagnosis can be achieved either with the use of cytological pictures viewed in real time from the microscope (dynamic telecytological systems) or with the use of cytological pictures that are first captured in a digital format and then transmitted using a storeand-forward approach to distant observers (static telecytological systems) [6-9]. In its simplest form, a static system comprises of a digital microscopic workstation comprising of microscope attached to a camera and a computer with high processing capacity and modem or internet connections. Digital images are transferred via file transfer protocol to specific password protected accounts, via secure hypertext protocol using a 40-bit encrypted server on the world wide web via an adaptable telepathology video management system or via Multipurpose Internet Mail Extensions (MIME)-encapsulated e-mail attachments [7-10]. Diagnoses made using telecytology should be as reliable as those made using conventional microscopy $[1,3]$. The existing studies which focused on the possible impact of static telecytology in the everyday laboratory's workflow have found a high concordance between telecytological and glass slide diagnoses [8-12]. However, little information exists about precaptured videos probable use for proficiency testing purposes [13]. Thus, the purpose of the article is to examine the feasibility of developing a proficiency-testing 
scheme for cytology labs wishing to be accredited according to ISO 15189:2012, by using precaptured videos.

\section{Materials and Methods}

The current study was carried out on 252 thyroid fine-needle aspiration specimens from 157 patients preoperatively aspirated under ultrasonographic guidance. For the purpose of this study, the 252 histologically confirmed cases were retrospectively selected from the department's registry, as well as copies of the original cytological and histological reports. The material collected was prepared by the ThinPrep2000 automated slide processor (CytycCo. [now Hologic, Bedford, MA]). From each case, one slide was prepared, stained by the Papanicolaou method, and examined by 3 independent board-certified cytopathologists. Conventional light microscopy was performed individually on an Olympus CX 31 microscope. Cytology reports were collected and statistically elaborated. The initial cytological diagnoses, made by conventional light microscopy, were made separately by each participant. In case of any major discrepancy, the final diagnosis was made after consultation and consensus of all participants. The initial cytological reports of each participant were kept for further statistical analysis (comparison with histology and measurement of intraobserver reproducibility between conventional cytology and telecytology made by precaptured videos). An other cytopathologist collected all 252-glass slides and captured 1 representative video from each case. Videos were captured with a Hamamatsu C4742-95 digital camera (Hamamatsu Photonics, Herrsching am Ammersee, Germany) mounted on an Olympus CX 31 microscope. The camera was connected via SCSI interface to a 1, 200-MHz Pentium CPU running Windows XP (Microsoft, Redmond, WA). Only one cytopathologist was assigned to capture 1 representative video from each case, in order to ensure homogenization of the criteria applied in field selection. The person appointed to capture representatives videos possessed adequate diagnostic experience and had not participated in the initial diagnostic round in order to avoid any bias concerning field selection. The videos captured were of 100 seconds duration each and provided valuable information that is routinely used in cytological diagnosis, such as background, cellularity, and nuclear and cytoplasmic details. More specifically, the criteria usually adopted for the selection of the captured areas were high cellularity, absence of obscuring factors, excellent preservation and visualization of nuclear and cytoplasmic details, background details (colloid), metaplastic changes, reactive cellular changes or cytological atypia (when present). The area covered by each video was about $20 \%$ of the slide area. All videos contained areas of both 100x and 400x magnification. The videos magnification was changing during video capture in order to focus to specific areas of interest. The intention of the study was to provide to all participating cytopathologists adequate digital material for making accurate diagnosis, without any methodological bias that could compromise the results of our study. All videos captured areas of interest at 100x and 400x magnifications with1, $124 \times 1,120$ resolution at 16-bit color depth and 300 dpi. 6, 12 and 24 months after videos capture, all 252 collected representative videos were transferred via file transfer protocol to specific password-protected accounts and were reviewed remotely by the same 3 cytopathologists on workstations using Google Chrome Web browser. All videos were accompanied by an electronic record of all crucial medical data that could have impact on the cytological diagnosis of all samples included in our study, such as patient age, relevant ultra-sonographic findings, thyroid hormonal status and past medical history. Reviewers commented on overall video quality using a 10-step scale, from 1 (very poor) to 10 (excellent). Reviewers had adequate experience to work with digital material and to use internet applications for diagnostic purposes. The diagnostic approach of precaptured videos concerning criteria of diagnosis, terminology of lesions, requirements of adequacy, and recommendations for further management were similar to those applied in conventional cytology (the 2017 Bethesda System for Reporting Thyroid Cytopathology) [14]. A detailed discussion of diagnostic criteria was not conducted between the cytopathologists, but all available clinical information was provided to all participants. Primarily, the cytological reports were classified in three basic diagnostic categories. "Benign", "malignant" and "suspicious" diagnoses were adopted. Diagnostic categories and their subclassification are presented in (Table 1). Cohen's $\kappa$ statistic was used for the calculation of intraobserver reproducibility. Mean intraobserver reproducibility was calculated as the weighted average of the individual kappa values. To determine the significance of the intraobserver kappa values, we used the Svanholm formula $[15,16]$. To assess interobserver agreement among cytopathologists for different diagnostic categories, kappa (j) statistics, first introduced in 1960, which provides a measure of agreement between two observers, was applied. Interpretation of kappa values is shown in (Table 2). Since our study involves more than two observers, a modification of kappa statistics allowing the comparison of more than two observers providing nominal (i.e. not ordered) answers, first proposed by Fleiss in 1971 was used $[15,16]$.

\section{Results}

Interobserver reproducibility among 3 cytopathologists using light microscopy is shown in (Table 3). Interobserver concordance among 3 cytologists who made the initial cytological diagnosis by light micros- copy is very high. The discordance percentage is negligible, so we can conclude that the 3 cytopathologists agree with each other $(\mathrm{p}<0.001)$. To confirm the reproducibility of the telecytology diagnosis and to study the interobserver agreement further, 6, 12 and 24 months after the first diagnostic round, the same representative digital videos were transferred in random order to the same cytopathologists and were reviewed again by them. The

Table 1: Diagnostic Results from conventional cytology.

\begin{tabular}{|c|c|}
\hline Diagnostic Categoriesv & Number of Cases \\
\hline Benign & 183 \\
\hline Nodular goiter-hyperplasia & 141 \\
\hline Thyroiditis & 42 \\
\hline Congenital abnormalities & 0 \\
\hline Malignant & 28 \\
\hline Papillary carcinoma & 26 \\
\hline Medullary carcinoma & 2 \\
\hline Intermediate & 41 \\
\hline Follicular neoplasm & 7 \\
\hline Oncocytic neoplasm & 3 \\
\hline Suspicious probably benign & 19 \\
\hline Suspicious probably malignant & 12 \\
\hline
\end{tabular}


Table 2: Interpretation of kappa values.

\begin{tabular}{|c|c|}
\hline$<0$ & Poor agreement \\
\hline $\mathbf{0 . 0}-0.20$ & Slight agreement \\
\hline $\mathbf{0 . 2 1 - 0 . 4 0}$ & Fair agreement \\
\hline $\mathbf{0 . 6 1 - 0 . 8 0}$ & Substantial agreement \\
\hline $\mathbf{0 . 8 1 - 1 . 0 0}$ & Almost perfect agreement \\
\hline
\end{tabular}

Table 3: Interobserver Reproducibility among 3 Cytopathologists Using Light Microscopy.

\begin{tabular}{|c|c|}
\hline Percent of overall agreement (P0) & 0.993704 \\
\hline Fixed-marginal k & 0.990121 \\
\hline Free-marginal k & 0.994022 \\
\hline
\end{tabular}

interobserver agreement was analyzed by using the diagnoses given by different cytopathologists on each one of the diagnostic rounds (Table 4). During the initial round, the diagnostic agreement was substantial among all cytopathologists. Interobserver agreement improved slightly during the following review rounds. Intraobserver reproducibility between light microscopy diagnoses and telecytology diagnoses was calculated among all cytopathologists with the corresponding overall kappa values ranging from 0.71 to 0.78 (Table 5). Cytological diagnoses based on digital videos and glass slides were compared with histological diagnoses. The investigation of intraobserver reproducibility among histological and telecytological diagnoses is presented in (Table 6). Diagnostic accuracy of diagnoses based on digital images and glass slides compared with post-thyroidectomy histological diagnosis is presented in (Table 7) and 8. Our results suggest that the level of concordance between telecytological and light microscopy diagnosis on a static telecytology consultation service (using pre-captured videos) may be as high as $85 \%$. The reviewers also commented on overall digital image quality. Reviewer's assessment of image quality is summarized in (Table 9).

\section{Discussion}

The slides collected for the production of the digital material from the Cytology Department's registry were coming from already histologically confirmed cases. The histological examination consists the best way cytology slides can be validated. Inadequate validation of test slides could lead to indiscriminate failure of qualified, competent personnel participating in external quality control programs [17-19]. To our knowledge the magnification used for the videos production
Table 6: Intraobserver Reproducibility Between static videos Telecytology and Histology.

\begin{tabular}{|c|c|c|c|}
\hline CYTOPATHOLOGIST & PA & PE & KAPPA (K) VALUE \\
\hline 1 & 0.84 & 0.39 & 0.74 \\
\hline 2 & 0.79 & 0.38 & 0.72 \\
\hline 3 & 0.82 & 0.38 & 0.7 \\
\hline Mean & & & 0.72 \\
\hline
\end{tabular}

PA, observed proportion of agreement; PE, proportion of agreement expected by chance.

Table 7: Diagnostic Accuracy of Diagnoses Based on Glass Slides Compared.

\begin{tabular}{|c|c|c|}
\hline $\begin{array}{c}\text { Conventional Vs. Histological } \\
\text { Diagnosis }\end{array}$ & Index & 95\% Confidence Interval \\
\hline Sensitivity & $89.47 \%$ & $85.61-93.34 \%$ \\
\hline Specificity & $98.53 \%$ & $97.01-100 \%$ \\
\hline Positive predictive value & $91.89 \%$ & $88.45-95.33 \%$ \\
\hline Negative predictive value & $98.05 \%$ & $96.31-99.79 \%$ \\
\hline Total accuracy & $97.11 \%$ & \\
\hline Prevalence & $15.70 \%$ & \\
\hline Unsatisfactory rate & $3.97 \%$ & \\
\hline
\end{tabular}

was not a contributing factor to high interobserver agreement. High video quality was crucial for correct telecytological diagnosis. The maximum quality of the captured videos was ensured by continuous monitoring of imaging variables such as sharpness, contrast, colors and magnification. In our study, the cytopathologist appointed to capture the representative videos from each slide had enough experience to select the most representative regions from each slide examined. The routine practice of telecytology may take place between a cytopathologist and another cytopathologist in a remote location [18-21]. Static telecytology systems capture cytologic pictures in a digital format followed by transmission to a distant observer. In its simplest form, a static system comprises of a digital microscopic workstation comprising of microscope attached to a camera and a computer with high processing capacity and modem or internet connections [18-21]. Digital images are transferred via file transfer protocol to specific password protected accounts, via secure hypertext protocol using a 40-bit encrypted server on the world wide web via an adaptable telepathology image management system or via Multipurpose Internet Mail Extensions (MIME)-encapsulated e-mail attachments $[1,3,18,19]$. The main disadvantages of static telepathology relate to the fact that the consulting telepathologist

Table 4: Interobserver reproducibility of static telecytology diagnoses for each diagnostic category.

\begin{tabular}{|c|c|c|c|}
\hline \multirow{2}{*}{$\begin{array}{c}\text { Diagnostic Categories (2017 Bethesda System for Reporting Thyroid } \\
\text { Cytopathology ) }\end{array}$} & \multicolumn{3}{|c|}{ KAPPA VALUE } \\
\hline & $1^{\text {st }}$ diagnostic round & $2^{\text {nd }}$ diagnostic round & $3^{\text {rd }}$ diagnostic round \\
\hline Diagnostic category I (Benign lesions) & 0.74 & 0.76 & 0.79 \\
\hline Diagnostic category II (Intermediate lesions) & 0.71 & 0.72 & 0.71 \\
\hline Diagnostic category III (Malignant lesions) & 0.76 & 0.78 & 0.79 \\
\hline
\end{tabular}

Table 5: Intraobserver Agreement Between Conventional Cytological and Telecytological Diagnoses (K-Values).

Table 5: Intraobserver Agreement Between Conventional Cytological and Telecytological Diagnoses (K-Values).
\begin{tabular}{|c|c|c|}
\hline \multirow{2}{*}{ Conventional Cytological Diagnosis Versus } & \multicolumn{2}{|c|}{ kappa value } \\
\cline { 2 - 4 } & $\mathbf{1}^{\text {st }}$ diagnostic round & $\mathbf{2}^{\text {nd }}$ diagnostic round \\
\hline Cytopathologist 1's telediagnosis & 0.72 & 0.74 \\
\hline Cytopathologist 2's telediagnosis & 0.71 & 0.73 \\
\hline Cytopathologist 3's telediagnosis & 0.74 & 0.76 \\
\hline
\end{tabular}


Table 8: Diagnostic Accuracy of Diagnoses Based on precaptured videos Compared with Histological Diagnosis (95\% Confidence Interval).

\begin{tabular}{|c|c|c|}
\hline Static Video Telecytology Vs. Histological Diagnosis & Cytopathologist 1 & Cytopathologist 2 \\
\hline Sensitivity & $92.11 \%$ & $86.84 \%$ \\
\hline Specificity & $98.53 \%$ & $98.53 \%$ \\
\hline Positive predictive value & $98.47 \%$ & $91.67 \%$ \\
\hline Negative predictive value & $91 \%$ & $97.57 \%$ \\
\hline Total accuracy & $98.53 \%$ & $96.69 \%$ \\
\hline Prevalence & $97.52 \%$ & $15.70 \%$ \\
\hline
\end{tabular}

Table 9: Estimation of videos quality.

\begin{tabular}{|c|c|c|c|}
\hline Image Quality & CYT 1 & CYT 2 & CYT 3 \\
\hline $\mathbf{1 0}$ & 220 & 203 & 213 \\
\hline $\mathbf{9}$ & 10 & 12 & 7 \\
\hline $\mathbf{8}$ & 4 & 9 & 8 \\
\hline $\mathbf{7}$ & 6 & 9 & 9 \\
\hline $\mathbf{6}$ & 6 & 6 & 7 \\
\hline $\mathbf{5}$ & 4 & 3 & 2 \\
\hline $\mathbf{4}$ & 1 & 4 & 4 \\
\hline $\mathbf{3}$ & 1 & 4 & 1 \\
\hline $\mathbf{2}$ & & 2 & 1 \\
\hline $\mathbf{1}$ & & & \\
\hline Total & 252 & 252 & 252 \\
\hline
\end{tabular}

has no remote control of the microscope or imaging device/ camera, their interpretation is limited to only captured field of views $[1,3,5,7]$. Static telecytology systems are used for evaluation of highly selected static images focusing only on few cells that may not show the entire evidence that is required for cytologic diagnosis [3,5,7,13]. Precaptured videos ensure continuity of cytological observation and provide substantial evidence of the representativeness of the selected fields $[3,5,7,13]$. The most common manifestation of interobserver discrepancy is upgrading of the telecytological diagnosis to a definitive carcinoma diagnosis or downgrading of a suspicious telecytological diagnosis to a rather benign lesion because of video deficiencies $[3,5,7,13]$. High digital video resolution and good color reproduction in this study contributed to the agreement of the three experienced cytopathologists. Cytology quality assessment is not very similar to clinical laboratory proficiency testing $[7,13,22,23]$. While clinical laboratory testing results are very dependent on instrument calibration and reagents, less frequent assessment is appropriate for the well-trained cytology professional who is assessing slides on a regular basis $[13,22]$. Since there is no evidence to suggest that cytology screening and interpretive abilities deteriorate after one year, we suggest that the suggested proficiency testing interval may be lengthened to two years rather than the current one year test interval. Telecytology by means of pre-captured videos, when integrated into the daily work flow, can provide special consultation opportunities to distant laboratories [24-26]. We suggest that static telecytology systems are preferred because of their low cost by laboratories that cannot afford the high cost of buying and maintaining dynamic systems. In addition, the telecommunication costs for the operation of static systems is lower as the usual common ADSL Internet connection is adequate to operate a static system. Criteria for interlaboratory static telecytological comparisons are not established yet, still there is enough evidence that an adequate number of alternative answers is available to participants, that participants must respond in the correct series at least $90 \%$ of the time and that the standard error of this percentage is less than $0.05[1,13]$. Besides histological examination, other measures for cytology slides validation should be adopted in other to avoid possible indiscriminate failure of qualified, competent personnel participating in external quality control programs. Such measures could be the verification of cytological diagnosis by board certified, well trained scientific personnel, the establishment of specific scoring system and reporting terminology for all kinds of cytological specimens and finally capturing of representative videos by certified well trained personnel [13]. Telecytology may be difficult to integrate into daily workflow $[1,7,13]$. Distant cytopathology departments with high needs for assistance in the form of expert consultation can rarely afford the high cost of buying and maintaining dynamic systems $[1,7,13]$. Static telecytological systems are affordable by all cytology departments and give the opportunity to all scientific personnel to participate in proficiency testing programs, even when there is a significant time difference among participating laboratories. Moreover, laboratory management should encourage personal participation of all scientific personnel in such proficiency testing programs $[1,7,13]$. Cytology scientific societies should focus on cytology proficiency testing particularities and define special technical aspects such as videos size and analysis, suggested testing intervals, diagnostic categories and methodology used for the statistical evaluation of the proficiency testing results $[1,7,13]$. Finally, proficiency testing providers should ensure that the personnel appointed to videos capture and transmission has adequate experience in both conventional and video-based diagnosis. Previous experience in that field should be well documented and recorded $[13,26,27]$. Last but not least, digital videos storage and transmission must follow strict regulations in order to avoid any unauthorized alteration or improper use. Current standards of electronic medical data handling are still informative, yet the need for a secure electronic environment, especially in the field of static telecytology, continues to grow $[13,27]$.

\section{References}

1. Archondakis S. The Use of Static Telecytology Applications for Accreditational Purposes. International Journal of Reliable and Quality E-Healthcare (IJRQEH). 2020; 9: 24-34.

2. Nagy GK, Newton LE. Comments on "Scientific issues related to the cytology proficiency testing regulations”. Acta Cytol. 2008; 52: 117-118.

3. Archondakis S, Roma M, Kaladelfou E. The Use of Static Telecytology for 
Quality Assessment Purposes in the Evaluation of Cervical Smears Prepared by Means of Liquid-Based Cytology. 2020; 26: 1522-1525.

4. Nagy GK, Newton LE. Review of Penner's criteria for proficiency testing in cytopathology: implications for the redesign of the federally mandated proficiency testing in cytopathology. Diagn Cytopathol. 2007; 35: 61-64.

5. Archondakis S, Georgoulakis J, Stamataki M, Anninos D, Skagias L, Panayiotides I, et al. Telecytology: a tool for quality assessment and improvement in the evaluation of thyroid fine-needle aspiration specimens. Telemed J E Health. 2009; 15: 713-717.

6. Raab SS, Zaleski MS, Thomas PA, Niemann TH, Isacson C, Jensen CS Telecytology: diagnostic accuracy in cervical-vaginal smears. Am J Clin Pathol. 1996; 105: 599-603.

7. Archondakis S, Roma M, Kaladelfou E. The Implementation of Static Telecytology for Teleconsultation Purposes during Preoperative Evaluation of Thyroid Fine-Needle Aspiration Specimens. 2020.

8. Pantanowitz L, Hornish M, Goulart RA. The impact of digital imaging in the field of cytopathology. Cytojournal. 2009; 6 : 6.

9. Pinco J, Goulart RA, Otis CN, Garb J, Pantanowitz L. Impact of digital image manipulation in cytology. Arch Pathol Lab Med. 2009; 133: 57-61.

10. Hanna MG, Monaco SE, Cuda J, Xing J, Ahmed I, Pantanowitz L. Comparison of glass slides and various digital-slide modalities for cytopathology screening and interpretation. Cancer Cytopathol. 2017; 125: 701-709.

11. Khurana KK. Telecytology and its evolving role in cytopathology. Diagn Cytopathol. 2012; 40: 498-502.

12. Tsilalis T, Archondakis S, Meristoudis C, Margari N, Pouliakis A, Skagias L, e al. Assessment of static telecytological diagnoses' reproducibility in cervical smears prepared by means of liquid-based cytology. Telemed J E Health. 2012; 18: 516-520.

13. Archondakis S. Remote Evaluation of Short Videos Captured by Static Telecytological Applications for Obtaining Expert Opinions. International Journal of Reliable and Quality E-Healthcare (IJRQEH), 2020; 9: 52-62.

14. Cibas ES, Ali SZ. The 2017 Bethesda System for Reporting Thyroid Cytopathology. Thyroid. 2017; 27: 1341-1346.

15. Koch GG, Landis JR, Freeman JL, Freeman DH Jr, Lehnen RC. A genera methodology for the analysis of experiments with repeated measurement of categorical data. Biometrics. 1977; 33: 133-158.

16. Landis JR, Koch GG. The measurement of observer agreement for categorical data. Biometrics. 1977; 33: 159-174.

17. Williams BH, Mullick FG, Butler DR, Herring RF, O'leary TJ. Clinical evaluation of an international static image-based telepathology service. Hum Pathol. 2001; 32: 1309-1317.

18. Chantziantoniou N, Mukherjee M, Donnelly AD, Pantanowitz L, Austin RM. Digital Applications in Cytopathology: Problems, Rationalizations, and Alternative Approaches. Acta Cytol. 2018; 62: 68-76.

19. Dalquen P, Savic Prince S, Spieler P, Kunze D, Neumann H, EppenbergerCastori $S$, et al. Making cytological diagnoses on digital images using the iPath network. Acta Cytol. 2014; 58: 453-460.

20. Thrall M, Pantanowitz L, Khalbuss W. Telecytology: Clinical applications, current challenges, and future benefits. J Pathol Inform. 2011; 2: 51.

21. Caron JE, Ying Y, Ye Q, Cheng L, Rao JY. International telecytology: Current applications and future potential. Diagn Cytopathol. 2019; 47: 28-34.

22. Lee ES, Kim IS, Choi JS, Yeom BW, Kim HK, Han JH, et al. Accuracy and reproducibility of telecytology diagnosis of cervical smears. A tool for quality assurance programs. Am J Clin Pathol. 2003; 119: 356-60.

23. Woodhouse SL, Schulte MA, Stastny JF. Proficient or deficient: on the razor's edge: establishing validity in cytology proficiency testing. Diagn Cytopathol. 1999; 20: 255-256.

24. García-Rojo M. International Clinical Guidelines for the Adoption of Digital Pathology: A Review of Technical Aspects. Pathobiology. 2016; 83: 99-109.

25. Nishat R, Ramachandra S, Behura SS, Kumar H. Digital cytopathology. J Oral Maxillofac Pathol. 2017; 21: 99-106.

26. Demichelis F, Della Mea V, Forti S, Dalla Palma P, Beltrami CA. Digital storage of glass slides for quality assurance in histopathology and cytopathology. J Telemed Telecare. 2002; 8: 138-142.

27. Nagy GK, Newton LE. Cytopathology proficiency testing: where do we go from here? Diagn Cytopathol. 2006; 34: 257-264. 